\title{
Pharmacogenomics Testing Confirmation of Carbamazepine Induced DRESS Reaction of an HLA-A*31:01 Positive, Polypharmacy Patient
}

\author{
Leigh Speicher, $M D^{1}$; Sheena Crosby, PharmD, BCGP2; Michael J. Schuh, PharmD, MBA, FAPhA \\ ${ }^{1}$ General Internal Medicine, Mayo Clinic Florida; Contribution: writing, clinical resource \\ ${ }^{2}$ Ambulatory Pharmacist, Mayo Clinic Florida; Contribution: writing, review \\ ${ }^{3}$ Family Medicine, and Palliative Medicine, Mayo Clinic Florida; Contribution: concept, writing, clinical resource, review, editing
}

\begin{abstract}
Pharmacogenomics (PGX) melds well with polypharmacy as another tool to identify medication related problems (MRPs) more specifically so they may be solved most effectively. PGx can pre-emptively assist in medication selection, medication dosing or identify better medications for patients already taking a medication. PGx can also confirm suspect medications of causing MRPs such as adverse drug reactions (ADRs) or drug interactions. In this case, PGx testing confirmed presence of a serious human leukocyte antigen (HLA) drug reaction with eosinophilia and systemic symptoms (DRESS) after a suspect medication had been stopped.
\end{abstract}

Keywords: pharmacogenomics, polypharmacy, collaboration, HLA, DRESS

\section{Introduction}

Pharmacogenomics (PGX) is a new science that lends itself well to the care of polypharmacy patients. ${ }^{1-2}$ Polypharmacy patients tend to be older, have more co-morbid conditions and because of this are also the medication use population more likely to suffer from medication related problems (MRPs) such as adverse drug reactions (ADRs) or drug interactions (DIs). ${ }^{3-6}$ A contributor to these MRPs may be PGx related via drug-gene interaction, drug-drug-gene interaction or drug-gene-gene interaction. PGx lab testing can be predictive, used for managing current therapy, preventative or in this case, assist with diagnosis and prevention of a serious PGx-related drug ADR. ${ }^{1-2}$

Human Leukocyte antigen (HLA) genes are responsible for drug reaction with eosinophilia and systemic symptoms (DRESS). ${ }^{7}$ DRESS reactions are more specifically diagnosed and categorized most commonly as Stevens-Johnson Syndrome (SJS) and Toxic Epidermal Necrosis (TEN)..$^{8-9}$ DRESS has been historically associated with antiepileptic drugs (AEDs) such as phenytoin, carbamazepine, oxcarbazepine, fosphenytoin and lamotrigine but may also be associated with allopurinol, abacavir and other non-antiepileptic drugs. ${ }^{10}$ DRESS can be fatal if the precipitating agent is not removed early enough. ${ }^{8}$

\section{Corresponding author:}

Michael J. Schuh, PharmD, MBA, FAPhA

Assistant Professor of Pharmacy

Family Medicine, and Palliative Medicine, Mayo Clinic Florida

4500 San Pablo Rd, Jacksonville, FL 32224

Work: 904-953-2673; Work fax: 904-953-2274

E-mail: Schuh.michael@mayo.edu

\section{Setting}

The setting is a tertiary care, destination medical clinic in Florida. The patient was seen in the General Internal Medicine (GIM) Department as an Executive Medicine patient. General Internal Medicine is a gateway medical practice for destination patients who seek further diagnostics, second or third opinions, further evaluation and diagnosis or tertiary care as complex internal medicine patients. The GIM physician acts as the gatekeeper for the patient care team and usually sees the patient referred from an outside provider first. They then internally refer the patient to different clinical departments for specialist consults, then sees the patient upon discharge from the clinic with a treatment plan. The plan considers all of the specialist consult opinions, including polypharmacy patients seen by medication therapy management (MTM) pharmacists, sometimes referred to as pharmacotherapy pharmacists, who routinely incorporate PGx into their practice.

\section{Case Report}

A 61-year-old female with a past medical history of supraventricular tachycardia (SVT), gastroesophageal reflux disease (GERD), irritable bowel syndrome (IBS), osteoporosis, allergic rhinitis, anxiety, migraines, and restless leg syndrome presented for an Executive Medicine physical exam. Seven months prior to her visit, she noted pain above her left eye and a rash that was diagnosed as herpes zoster. She had been working with a home neurologist for ongoing pain consistent with post-herpetic neuralgia. After prednisone and increased doses of gabapentin failed, she was started on carbamazepine 5 weeks prior to her presentation. She did find this helpful for the pain, but it had not resolved. Five days prior to the medical visit, she went to the ER for low back pain and fever. She had a computed tomography (CT) scan of the abdomen and labs. She states that the urine test was not conclusive but showed a small amount of blood. No other abnormalities were discussed. She was told that they would treat her for 
urinary tract infection (UTI) that may be evolving into pyelonephritis. She was given a 10-day course of cephalexin. During her evaluation, she described a new tremor in her hands and unsteady gait. She noted bumps on her legs that started 2 weeks prior. The day prior to her visit, a red, nonpruritic rash spread to her trunk and extremities. She stopped the cephalexin and the carbamazepine immediately when the rash developed.

On exam, her vital signs were within normal limits. Her physical exam was significant for a diffuse maculopapular rash and bilateral resting tremor of the hands. Labs were significant for an elevated alanine aminotransferase (ALT) of 543 (Ref: 7$45 \mathrm{U} / \mathrm{L}$ ), aspartate aminotransferase (AST) of 200 (Ref: 8-43 $\mathrm{U} / \mathrm{L}$ ), and alkaline phosphatase of 354 (Ref 35-104 U/L). Her bilirubin and albumin were normal. Her international normalized ratio (INR) was slightly elevated at 1.3 (Ref: 0.91.1). Eosinophils were elevated at $13.5 \%$ (Ref: $1-3 \%$ ) and 0.80 (Ref: 0.03-0.48x10(9)/L). Her urinalysis did show leukocyte esterase with negative nitrites and 3 red blood cells per highpowered field. Liver function tests (LFTs) at our facility which had been checked annually over the past 8 years had been normal.

On her day of presentation, she was seen in hepatology and dermatology. Hepatology noted her that her ALT was greater than 10 times normal and alkaline phosphatase was greater than 3 times normal. They felt that the minimal elevation in INR was not significant as there were no other hematologic abnormalities besides a slight eosinophilia consistent with a drug reaction. She denied alcohol intake, risk factors for viral hepatitis, and autoimmune disease. She was diagnosed with acute drug related hepatic injury due to carbamazepine since cephalexin is not known to commonly elevate LFT's. Additional labs were conducted and showed no evidence of other underlying liver disease. As the outside CT from the ER had not yet been obtained, an ultrasound of the liver was performed the next day. It demonstrated mildly decreased liver echogenicity which can be due to edema in the setting of acute hepatitis. There was no sign of chronic liver disease. Recommendation was made to monitor LFT's to normalization and return if they remained stable or increased. Dermatology diagnosed her with a morbilliform drug eruption. They noted that the likely offending agent had been stopped and recommended gentle skin cleansers and moisturizers. Carbamazepine was also added to her allergy list.

She returned just over 1 month later for repeat labs, an evaluation in neurology, and an evaluation our pharmacotherapy clinic. Her LFT's normalized upon testing during this visit. In neurology, she was noted to have ongoing hypersensitivity to touch over her left medial forehead in the distribution of the prior rash. She also had localized pain over the superior medial canthus of her left eye at the origin of the supratrochlear nerve. She was diagnosed with left supratrochlear neuropathic pain secondary to post herpetic neuralgia and was started on amitriptyline. This was titrated up and this now controls her pain. During her pharmacist, pharmacotherapy appointment, a PGx consultation with labs was recommended by the pharmacist.

PGx lab testing with pharmacist consult were then ordered by the attending physician. PGx lab results on follow up indicated patient was HLA-A*31:01 positive, suggesting rash, eosinophilia and elevated LFTs were due to DRESS hypersensitivity to the carbamazepine. After the positive HLA test, carbamazepine was then listed in the patient's electronic medical record (EMR) as a gene-drug intolerance under allergies and cross-referenced through the EMR to produce a clinical decision alert if carbamazepine was every ordered.

\section{Discussion}

PGx panel testing can assist in predicting what medications to avoid, eliminating the trial and error method of medication selection. In this case testing was used to confirm a diagnosis even though the precipitating agent was stopped. HLA genes, responsible for regulation of immune response, may be ordered separately and are not on all PGx lab panels.

HLA genes are many but those tested for most are HLAA*31:01, HLA-B*58:01, HLA-B*57:01 and HLA-B*15:02 corresponding with hypersensitivities to allopurinol, abacavir and many antiepileptic drugs, including carbamazepine.

DRESS symptoms may not appear right away in an HLA sensitive patient. ${ }^{11}$ The latency phase between start of medication and symptoms range from 2 to 8 weeks. ${ }^{11}$ Not all patients who are sensitive will exhibit DRESS symptoms, some may express them differently and DRESS symptoms may appear as minor but progress quickly to a severe, life threatening dermatological condition. Skin manifestations are often first noticed on the trunk and extremities. Other symptoms are fever, eosinophilia, malaise and elevated LFTs. This patient exhibited eosinophilia, elevated LFTs and rash. Certain subpopulations are more prone to DRESS such as certain southeast Asian populations. So much so, HLA testing is mandatory before certain medications such as carbamazepine can be administered in these subpopulations. ${ }^{12}$

In this case causality was associated with a start of a known medication to cause specific symptoms, resolution after the agent was withdrawn then objective PGx testing to verify patient sensitivity to the agent, carbamazepine. ${ }^{13-14}$ This patient scored an " 8 " on the Naranjo Adverse Drug Reaction Probability Scale, indicating that the rash was associated with the administration of the carbamazepine. (Tables I \& II) The Naranjo Scale is used as an objective tool to determine degree of cause and effect pertaining to ADRs. 


\section{Conclusion}

Much of the time PGx is used in predictive or current treatment to assist with the most suitable medications with regard to medication management. This case demonstrates PGx testing may also be used to help retrospectively confirm a suspected ADR to avoid a medication or related medications in the future that may produce the same potentially serious or life-threatening ADR. The case also demonstrates how physicians and pharmacists can work collaboratively using a new science to help produce better patient outcomes.

The authors have no conflicts of interest or financial ties to disclose.

The opinions contained in the paper are those of the authors.

\section{References}

1. Liu J, Friedman C, Finkelstein J. Pharmacogenomic Approaches for Automated Medication Risk Assessment in People with Polypharmacy. AMIA Jt Summits Transl Sci Proc. 2018;2017:142-151. Published 2018 May 18.

2. Finkelstein J, Friedman C, Hripcsak G, Cabrera M. Potential utility of precision medicine for older adults with polypharmacy: a case series study. Pharmgenomics Pers Med. Published 2016 Apr 15. 2016;9:31-45.

3. Avorn J, Gurwitz JH. Drug use in the nursing home. Ann Intern Med. 1995 Aug 1;123(3):195-204.

4. Masnoon N, Shakib S, Kalisch-Ellett L, et al. What is polypharmacy? A systematic review of definitions. BMC Geriatr 2017;17: 230.

5. Maher RL, Hanlon J, Hajjar ER. Clinical consequences of polypharmacy in elderly. Expert Opin Drug Saf. 2014;13(1):57-65.

6. Kojima G, Bell C, Tamura B, et al. Reducing cost by reducing polypharmacy: the polypharmacy outcomes project. J Am Med Dir Assoc. 2012;13(9):818.e11-818.e8.18E15.

7. McCormack M, Alfirevic BA, Bourgeois S, Farrell JJ, et all. HLA-A*3101 and Carbamazepine-Induced Hypersensitivity Reactions in Europeans. N Engl J Med 2011; 364:1134-1143.

8. De A, Rajagopalan M, Sarda A, Das S, Biswas P. Drug Reaction with Eosinophilia and Systemic Symptoms: An Update and Review of Recent Literature. Indian J Dermatol. 2018;63(1):30-40.

9. Genin, E, Chen, DP, Hung, SI. et al. HLA-A*31:01 and different types of carbamazepine-induced severe cutaneous adverse reactions: an international study and meta-analysis. Pharmacogenomics J 14, 281-288 (2014).

10. Whirl-Carrillo M, McDonagh EM, Hebert JM, Gong L, Sangkuhl K, Thorn CF, Altman RB and Klein TE. Pharmacogenomics Knowledge for Personalized Medicine. Clinical Pharmacology \& Therapeutics (2012) 92(4): 414-417.

11. Soria A, Bernier C, Veyrac G, Barbaud A, Puymirat E, Milpied B. Drug reaction with eosinophilia and systemic symptoms may occur within 2 weeks of drug exposure: A retrospective study J Am Acad Dermatol. 2020;82(3):606. Epub 2019 Sep 25.

12. Yip V, PirmohamedM. The HLA-A*31:01 allele: influence on carbamazepine treatment.

Pharmgenomics Pers Med. 2017; 10: 29-38. Published online 2017 Jan 31.

13. Rehan HS, Deepti C, Kakkar AK. Physician's guide to pharmacovigilance: terminology and causality assessment. Eur J Intern Med 2009;20:3-8.

14. U. S. National Library of Medicine Livertox Database. Available at http://livertox.nih.gov/Narajo.html. Accessed March 20, 2021. 
Table I: Naranjo Adverse Drug Reaction (ADR) Probability Scale

\begin{tabular}{|c|c|c|c|c|}
\hline Question & Yes & No & $\begin{array}{l}\text { Do Not } \\
\text { Know }\end{array}$ & Score \\
\hline 1. Are there previous conclusive reports on this reaction? & +1 & 0 & 0 & 1 \\
\hline 2. Did the adverse event appear after the suspected drug was administered? & +2 & -1 & 0 & 2 \\
\hline $\begin{array}{l}\text { 3. Did the adverse event improve when the drug was discontinued or a specific antagonist was } \\
\text { administered? }\end{array}$ & +1 & 0 & 0 & 1 \\
\hline 4. Did the adverse event reappear when the drug was readministered? & +2 & -1 & 0 & $\mathrm{n} / \mathrm{a}^{*}$ \\
\hline 5. Are there alternative causes that could on their own have caused the reaction? & -1 & +2 & 0 & 2 \\
\hline 6. Did the reaction reappear when a placebo was given? & -1 & +1 & 0 & $\mathrm{n} / \mathrm{a}^{*}$ \\
\hline 7. Was the drug detected in blood or other fluids in concentrations known to be toxic? & +1 & 0 & 0 & 0 \\
\hline $\begin{array}{l}\text { 8. Was the reaction more severe when the dose was increased or less severe when the dose was } \\
\text { decreased? }\end{array}$ & +1 & 0 & 0 & 1 \\
\hline 9. Did the patient have a similar reaction to the same or similar drugs in any previous exposure? & +1 & 0 & 0 & 0 \\
\hline 10. Was the adverse event confirmed by any objective evidence? & +1 & 0 & 0 & 1 \\
\hline & \multicolumn{3}{|c|}{ Total Score: } & 8 \\
\hline
\end{tabular}

References $^{14-15}$

*n/a = Not applicable

Table II: Naranjo Algorithm - ADR Probability Scale

\begin{tabular}{|l|l|}
\hline Score & Interpretation of Scores \\
\hline Total Score $\mathbf{2 9}$ & $\begin{array}{l}\text { Definite. The reaction (1) followed a reasonable temporal sequence after a drug or in which a toxic drug level } \\
\text { had been established in body fluids or tissues, (2) followed a recognized response to the suspected drug, and } \\
\text { (3) was confirmed by improvement on withdrawing the drug and reappeared on reexposure. }\end{array}$ \\
\hline Total Score $\mathbf{5}$ to $\mathbf{8}$ & $\begin{array}{l}\text { Probable. The reaction (1) followed a reasonable temporal sequence after a drug, (2) followed a recognized } \\
\text { response to the suspected drug, (3) was confirmed by withdrawal but not by exposure to the drug, and (4) } \\
\text { could not be reasonably explained by the known characteristics of the patient's clinical state. }\end{array}$ \\
\hline Total Score $\mathbf{1}$ to $\mathbf{4}$ & $\begin{array}{l}\text { Possible. The reaction (1) followed a temporal sequence after a drug, (2) possibly followed a recognized } \\
\text { pattern to the suspected drug, and (3) could be explained by characteristics of the patient's disease. }\end{array}$ \\
\hline Total Score $\leq \mathbf{0}$ & Doubtful. The reaction was likely related to factors other than a drug. \\
\hline
\end{tabular}

References $^{13-14}$ 